\title{
An eHARS Dashboard for State HIV Surveillance
}

\author{
Elliott S. Brannon ${ }^{\star 1}$, Tera Reynolds ${ }^{2,3}$, Sunyang $\mathrm{Fu}^{2,3}$, Kristin Harden ${ }^{3}$, Dolorence \\ Okullo $^{2,3}$ and Shreyas Ramani ${ }^{2,3}$
}

${ }^{1}$ University of Michigan Medical School, Ann Arbor, MI, USA; ${ }^{2}$ University of Michigan School of Information, Ann Arbor, MI, USA;

${ }^{3}$ University of Michigan School of Public Health, Ann Arbor, MI, USA

\section{Objective}

Describe the development process and function of a data dashboard for state HIV surveillance and discuss the benefits of creating interactive data dashboards in the $\mathrm{R}$ software environment.

\section{Introduction}

State HIV offices routinely produce fact sheets, epidemiologic profiles, and other reports from the eHARS (Enhanced HIV/AIDS Reporting System) database which was created and is maintained by the CDC. ${ }^{1}$ The eHARS software is used throughout the United States to monitor the HIV epidemic and evaluate HIV prevention programs and policies. Due to limited variability of eHARS throughout the United States, software developed to analyze and visualize data using the eHARS database schema may be useful to many state HIV offices. Software developed based on the eHARS database schema could reduce the time required for analysis and production of reports.

The R software environment for statistical computing is an open source project with a thriving community of users who continue to expand R's analysis capacity through the addition of packages. A package is "a standardized collection of material extending R, e.g. providing code, data, or documentation". ${ }^{2}$ Shiny is one example of a user-developed package which easily allows $\mathrm{R}$ users to create interactive web applications from analytical software.

\section{Methods}

An open source R package is under development for state HIV offices. The package is being developed in the open on the Github repository. The eharsDash package will create a data dashboard based on the eHARS database schema and will rely on the Shiny package to create interactive data visualizations from the data in eHARS. The eharsDash package will also contain software to import data from eHARS into R for analysis and visualization.

Once developed, state HIV offices may use the software in several ways including the following:

1) Users may install the software locally for individual analyses

2) Offices may install the software on an intranet for use by multiple users in the office

3) Offices may install the the software on a publicly available website, allowing the public to interact with HIV data

Developers will work with state HIV offices to determine the visualization needs of the office and will also create plots based on HIV epidemiological profiles available from the websites of state HIV offices. If state HIV offices find the eharsDash package useful, the number of data visualizations can easily be expanded through the continuation of the community-driven process. State HIV offices and the CDC could provide feedback and work together with the developers to create an open-source data visualization package for the eHARS database.

\section{Conclusions}

The $\mathrm{R}$ software environment will used to create a powerful data dashboard for the eHARS database schema. The eharsDash package will contain software which imports data from eHARS into the R environment and analyzes and visualizes the data. It will also enable reproducibility of analyses. Due to this reproducibility, the use of the
eharsDash package may reduce the time state HIV offices require to complete analyses for reports. State HIV offices may then have additional resources to pursue research and prevention activities and reduce the burden of HIV in their state.

The eharsDash package will also encourage a more open structure for presenting HIV surveillance data while maintaining anonymity. Although traditionally state health departments have relied on SAS software for analysis and data management, consideration should be given to the $\mathrm{R}$ software environment due to its open environment, user community, and cost. Data transparency and access are essential to understanding and reducing the health inequalities which exist in diseases such as HIV.

\section{Keywords}

eHARS; Shiny; Visualization

\section{References}

1. HIV Open Data Project: eHARS - The HIV/AIDS Reporting System, Person Data (ICPSR 34725). http://doi.org/10.3886/ICPSR34725.v1. 2. R Development Core Team (2015). An Introduction to R.

URL http://www.R-project.org/manuals.html.

3. 2013 STD/HIV Program Report. State of Louisiana Department of Health and Hospitals, Office of Public Health.

4. 2014 STD/HIV Program Report. State of Michigan Department of Health and Hospitals, Office of Public Health.

\section{*Elliott S. Brannon}

E-mail: ebrann@umich.edu 